\title{
Fluidizing and Solidifying Effects of Perfluorooctylated Fatty Alcohols on Pulmonary Surfactant Monolayers
}

\author{
Hiromichi Nakahara* \\ Department of Biophysical Chemistry, Graduate School of Pharmaceutical Sciences, Nagasaki International University; 2825-7 Huis Ten Bosch, \\ Sasebo, Nagasaki 859-3298, JAPAN
}

\begin{abstract}
Pulmonary surfactant (PS) preparations based mainly on bovine or porcine extracts are commonly administered to patients with neonatal respiratory distress syndrome (NRDS) for therapy. The preparations are sufficiently effective to treat NRDS; however, they are associated with a risk of infection and involve costly purification procedures to achieve batch-to-batch reproducibility. Therefore, we investigated the mechanism and interfacial behavior of synthetic PS preparations containing a mimicking peptide (KLLKLLLKLWLKLLKLLL, Hel 13-5). In particular, a hybrid PS formulation with fluorinated amphiphiles is reported from the perspective of surface chemistry. Fluorinated amphiphiles are characterized by exceptional chemical and biological inertness, high oxygen-dissolving capacity, low surface tension, excellent spreading ability, and high fluidity. These properties are superior to those for the corresponding hydrocarbon analogs. Indeed, a small amount of fluorinated long-chain alcohols enhances the effectiveness of the model PS preparation for in vitro pulmonary functions. Moreover, the mode of the improved efficacy differs depending on the hydrophobic chain length in the alcohols. For alcohols with a short fluorocarbon (FC) chain, the monolayer phase of the model PS preparation remains disordered (fluidization). However, the addition of alcohols containing a long FC chain reduces the disordered/ordered phase transition pressure and the growth of ordered domains of the monolayer (condensation). Furthermore, repeated compression-expansion isotherms of the monolayers, which can simulate respiration in the lung, suggest irreversible elimination of the short-FC alcohol into the subphase and enhancement of the squeeze-out phenomenon of certain PS components by solid-like monolayer formation induced by the long-FC alcohol. We demonstrated that fluorinated amphiphiles may be used as additives for synthetic or commercial PS preparations for RDS treatment.
\end{abstract}

Key words: Langmuir monolayer, fluorinated amphiphile, DPPC, SP-B, pulmonary surfactant

\section{Introduction}

Perfluorinated chains ( $F$-chains) differ to large extents from hydrogenated chains ( $H$-chains) in terms of bulkiness (cross sections of $\sim 0.28$ and $0.18 \mathrm{~nm}^{2}$, respectively), helical conformation (all-trans, rather than planar 'zigzag' structure), and stiffness ${ }^{1)} . F$-chains are considerably more hydrophobic than $H$-chains but are also lipophobic ${ }^{2)}$. The low polarizability of fluorine (c.a. 0.557) generates very weak van der Waals interactions between $F$-chains ${ }^{3)}$. In addition, the fluorine atoms in $F$-chains do not commonly engage in hydrogen bonding ${ }^{4)} . F$-chains are also chemically and thermally more stable than $H$-chains. The properties of fluorinated amphiphiles or surfactants result in lower surface tension and higher vapor pressure than the corresponding hydrogenated molecules. In this regard, compounds containing $F$-chains have been synthesized as partially fluorinated compounds and their properties with respect to the degree of fluorination in a molecule have been extensively investigated ${ }^{5-9)}$. We previously studied fluorinated long-chain alcohols $\left(\mathrm{CF}_{3}\left(\mathrm{CF}_{2}\right)_{n-1}\left(\mathrm{CH}_{2}\right)_{m} \mathrm{OH}\right.$, $F n H m \mathrm{OH}$ ) because of the simplicity of their chemical structure (Fig. 1 ${ }^{10-14)}$.

\footnotetext{
*Correspondence to: Hiromichi Nakahara, Department of Biophysical Chemistry, Graduate School of Pharmaceutical Sciences, Nagasaki International University; 2825-7 Huis Ten Bosch, Sasebo, Nagasaki 859-3298, JAPAN

E-mail: nakahara@niu.ac.jp

Accepted November 10, 2015 (received for review October 6, 2015)

Journal of Oleo Science ISSN 1345-8957 print / ISSN 1347-3352 online

http://www.jstage.jst.go.jp/browse/jos/ http://mc.manusriptcentral.com/jjocs

This is the review by the winner of 49th Award for Prominent Studies, The Japan Oil Chemists' Society (JOCS).
} 


\section{H. Nakahara}

(a)

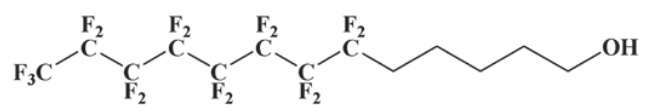

(b)

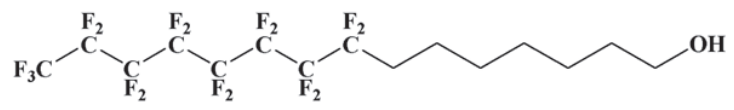

(c)

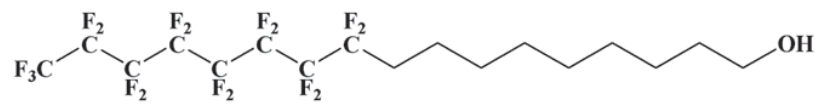

(d)

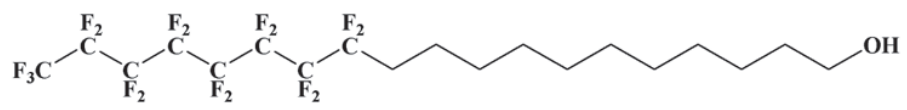

(e)

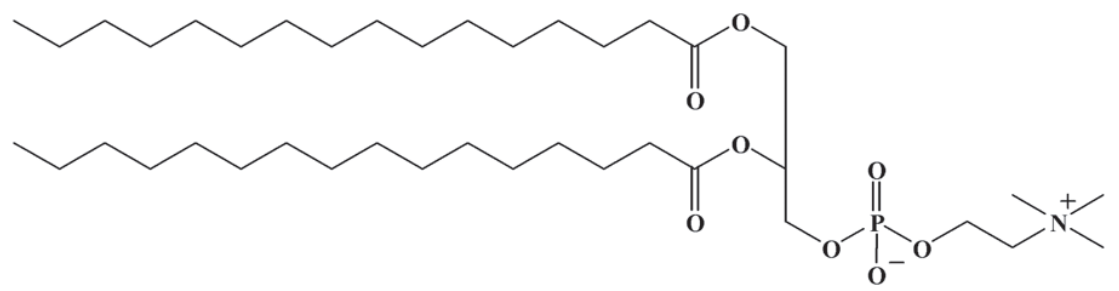

Fig. 1 Chemical structures of (a) $F 8 H 5 \mathrm{OH}$, (b) $F 8 H 7 \mathrm{OH}$, (c) $F 8 H 9 \mathrm{OH}$, (d) $F 8 H 11 \mathrm{OH}$, and (e) DPPC.

A pulmonary surfactant (PS) is a lipid-protein complex composed mainly of phospholipids such as dipalmitoylphosphatidylcholine (DPPC) and phosphatidylglycerol ${ }^{15-17}$. It plays an essential role in pulmonary function by reducing the surface tension of the alveoli ${ }^{18}$. The PS function has been summarized well in review articles ${ }^{18-22)}$. Surfactant proteins (SP-A, SP-B, SP-C, and SP-D) are present at relatively low levels in PS but are quite important for the control of lipid components at the surface ${ }^{21}$. SP-B and SP-C are critical for surface activity because of their strong hydrophobicity and low solubility in water ${ }^{17)}$. A native lack of PS causes neonatal respiratory distress syndrome (NRDS). As an artificial PS preparation, Surfacten ${ }^{\circledR}$, a bovine pulmonary surfactant extract supplemented with DPPC, palmitic acid (PA), and tripalmitin, has been used as a first-line treatment for NRDS in Japan. However, although it is effective for treating NRDS patients, Surfacten ${ }^{\circledR}$ may cause anthropozoonosis. In this regard, preparations for PS replacement treatments of NRDS patients have been extensively developed using no animal sources ${ }^{23-27)}$. We previously reported that PS preparations containing an amphiphilic peptide (Hel 13-5 or a mimicking peptide of SP-B) exert pulmonary activities and functions ${ }^{28-30}$. Hel 13-5 has been extensively characterized using various techniques such as circular dichroism spectroscopy, electron microscopy, infrared spectroscopy, etc. ${ }^{31-36)}$. These investigations showed that Hel 13-5 is a membrane-surface peptide that is similar to SP-B.

This article reviews recent evaluations of the effect of partially fluorinated alcohols $(F 8 \mathrm{HmOH})$ on model PS preparations consisting of DPPC and Hel 13-5. The fluorinated molecules are not macromolecules and thus show high thermal stability and antigen recognition related to biophylaxis. In particular, their thermal stability (or resistance) is considered a great advantage in terms of long-time storage and high-volume production. Currently, the application of air saturated with fluorocarbon gases for RDS treatments has been used ${ }^{37,38)}$ because of the properties of fluorocarbon such as low intermolecular cohesiveness, high vapor pressures relative to the molecular weights ${ }^{2)}$, and, in particular, high-dissolving oxygen capacity. A predominant symptom of RDS patients is dyspnea caused by the dysfunction and inactivation of PS; thus, partial ventilation with air and fluorocarbon gases is considered useful for treating RDS. However, the application is still under development because of the difficulty in controlling the partial pressures of the gases, which strongly depends on the state of the lungs. To understand the fundamental interfacial properties of the PS preparations, a monolayer technique was employed at the air-water interface. First, the miscibility and interaction between DPPC and $\mathrm{F} 8 \mathrm{HmOH}$ were systematically clarified within a monolayer. Next, we 
evaluated the potential use of partially fluorinated amphiphiles for application in synthetic PS preparations.

\section{Monolayer properties of DPPC and $\mathbf{F} \mathbf{8 m O H}$}

The surface pressure $(\pi)$-molecular area $(A)$ and the surface potential $(\Delta V)-A$ isotherms of $F 8 H m \mathrm{OH}(m=5,7$, 9 , and 11) and DPPC monolayers on $0.15 \mathrm{M} \mathrm{NaCl}$ are shown in Fig. 2. At $298.2 \mathrm{~K}, \mathrm{~F} 8 \mathrm{H} 5 \mathrm{OH}$ shows difficulty in forming an insoluble (or stable) monolayer at the air-water interface because of its high solubility in water ${ }^{12,14)}$. The $\pi-A$ isotherm of the $F 8 H 5 \mathrm{OH}$ monolayers (curve 1) showed a kink at $\sim 5 \mathrm{mN} \mathrm{m}^{-1}$, indicating a phase transition of the disordered state to the ordered state. The kink is much more obscure compared to that of a typical hydrogenated lipid such as DPPC (curve 5 ). However, the transition at $\sim 5 \mathrm{mN}$ $\mathrm{m}^{-1}$ has been confirmed by the observation that the disordered/ordered transition $\left(\pi^{\mathrm{eq}}\right)$ of $\mathrm{F} 8 \mathrm{H} 5 \mathrm{OH}$ monolayers increases linearly with increasing temperatures ${ }^{39)}$. The other $\mathrm{F} 8 \mathrm{Hm} \mathrm{OH}$ monolayers exhibited an isotherm of the typical ordered phase without such a transition. The collapse pressure $\left(\pi^{c}\right)$ of $F 8 H m \mathrm{OH}$ monolayers at which the monolayer was transformed into a bilayer or other three-dimensional structures increases slightly with increasing total chain lengths. Their limiting (or extrapolated) molecular

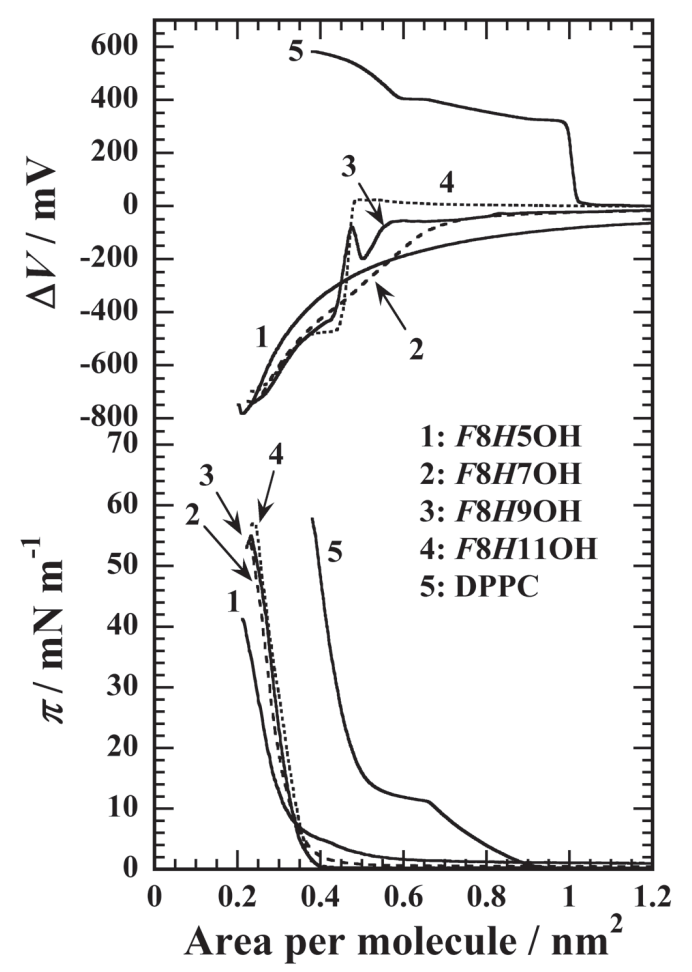

Fig. 2 The $\pi-A$ and $\Delta V-A$ isotherms of $F 8 H \mathrm{mOH}(\mathrm{m}=$ $5,7,9$, and 11) and DPPC monolayers on 0.15 $\mathrm{M} \mathrm{NaCl}$ at $298.2 \mathrm{~K}(293.2 \mathrm{~K}$ for $F 8 H 5 \mathrm{OH})$. areas are concentrated near $0.33-0.35 \mathrm{~nm}^{2}$, which is similar to the cross-sectional area of an $F$-chain $(\sim 0.30$ $\left.\mathrm{nm}^{2}\right)^{2)}$. Considering the cross-sectional area of an $H$-chain $\left(\sim 0.20 \mathrm{~nm}^{2}\right)$, the $F 8$-moiety in $F 8 H \mathrm{HOH}$ molecules dominates over the monolayer ordering and orientation in the close-packed state. In contrast, DPPC forms a typical liquid-expanded (LE)/liquid-condensed (LC) monolayer under the present conditions. The extrapolated area of the DPPC monolayers indicates a value of more than $0.40 \mathrm{~nm}^{2}$ (equally two $H$-chains) resulting from the lower ordering of two aliphatic chains even upon compression, which is induced by the bulkiness of the PC head group.

The $\Delta V-A$ isotherm of lipid monolayers consisting of $H$ chains or $F$-chains commonly exhibits positive (e.g. DPPC) or negative variation (e.g. $F 8 H m \mathrm{OH}$ ), respectively, with decreasing $A$. The $\Delta V$ value of DPPC monolayers remains constant $(\Delta V \approx 0)$ in the region of the gaseous phase because of the non-polarity of a molecule to the surfacenormal direction. As molecule areas decrease to below $A=$ $\sim 1.0 \mathrm{~nm}^{2}$, the $\Delta V$ value increases from 0 to $\sim 550 \mathrm{mV}$. In contrast, for $F 8 H m \mathrm{OH}$ monolayers, minimum $\Delta V$ (or $\Delta V_{\text {min }}$ ) values at the close-packed state reach approximately $-750 \mathrm{mV}$ independently of total chain lengths in $\mathrm{F} 8 \mathrm{Hm} \mathrm{OH}$. Thus, the close-packed states of $\mathrm{F} 8 \mathrm{HmOH}$ monolayers are similar to each other. The convergence of the $\Delta V_{\min }$ value and negative $\Delta V$ variation upon compression reveals that the terminal $\mathrm{CF}_{3}$ - group is exposed to air.

\section{Miscibility between DPPC and $\mathrm{F} 8 \mathrm{HmOH}$ monolayers}

Two-dimensional phase diagrams $\left(\pi\right.$ versus $\left.X_{F 8 H \mathrm{OH}}\right)$ are constructed based on the $\pi^{\mathrm{eq}}$ and $\pi^{\mathrm{c}}$ values for the twocomponent DPPC/F8HmOH monolayers. The phase diagrams of the DPPC/F8HmOH systems are shown in Fig. 3. All systems here indicate changes in $\pi^{\mathrm{eq}}$ with respect to $X_{F 8 \mathrm{Hm} \mathrm{OH}}$, suggesting the miscibility between DPPC and $F 8 H m \mathrm{OH}$ in the monolayer state. The DPPC/F8H5OH system shows convex variation in $\pi^{\mathrm{eq}}$ against $X_{F 8 H 50 \mathrm{H}}$. However, for the other systems, the $\pi^{\text {eq }}$ value decreases with increasing $X_{F 8 H \mathrm{OH}}$ and the slope of $\partial \pi^{\mathrm{eq}} / \partial X_{F 8 \mathrm{HmOH}}$ becomes steeper as total chain lengths are elongated in $\mathrm{F} 8 \mathrm{HmOH}$. It was found that from these $\pi^{\mathrm{eq}}$ changes, the addition of $\mathrm{F} 8 \mathrm{H} 5 \mathrm{OH}$ or $\mathrm{F} 8 \mathrm{HmOH}(m \geq 7)$ produces fluidizing or solidifying effects on DPPC monolayers, respectively. Additionally, the extension of the methylene group $\left(-\mathrm{CH}_{2}-\right.$ ) in the alcohols may promote the solidifying effect.

The coexistence of a phase boundary between the twodimensional/three-dimensional phases of the film-forming molecules spread on a surface can be theoretically simulated using the Joos equation ${ }^{40,41)}$ under the assumption of a regular surface mixture: 


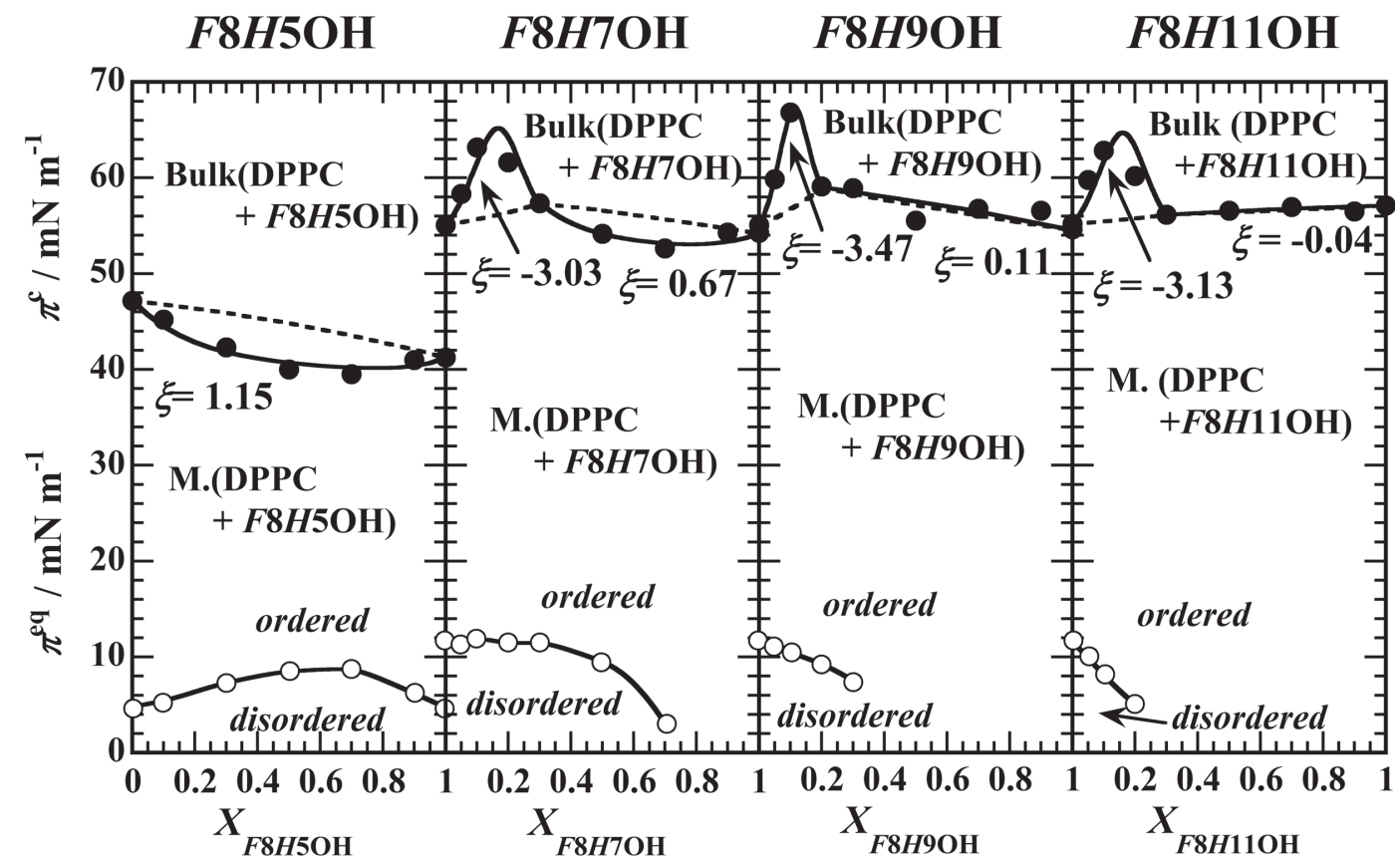

Fig. 3 Two-dimensional phase diagrams based on the variation of the transition pressure ( $\pi^{\text {eq. }}$ : open circle) and collapse pressure $\left(\pi^{\mathrm{c}}\right.$ : solid circle) for the two-component DPPC/F8HmOH systems on $0.15 \mathrm{M} \mathrm{NaCl}$ at $298.2 \mathrm{~K}(293.2 \mathrm{~K}$ for the $F 8 H 5 \mathrm{OH}$ system) as a function of $X_{\mathrm{F} 8 \mathrm{Hm} \mathrm{OH}}$. In the high surface pressure region, the dashed lines were calculated according to Eq. (1) for $\xi=0$. The solid line was obtained by curve fitting of experimental collapse pressures to Eq.(1). M. indicates a mixed monolayer formed by DPPC and $\mathrm{F} 8 \mathrm{HmOH}$ species, whereas Bulk denotes a solid phase of the two components.

$$
\begin{aligned}
1= & x_{1}^{\mathrm{s}} \exp \left[\left(\pi_{\mathrm{m}}^{\mathrm{c}}-\pi_{1}^{\mathrm{c}}\right) \omega_{1} / k T\right] \exp \left[\xi\left(x_{2}^{\mathrm{s}}\right)^{2}\right]+x_{2}^{\mathrm{s}} \exp \left[\left(\pi_{\mathrm{m}}^{\mathrm{c}}-\right.\right. \\
& \left.\left.\pi_{2}^{\mathrm{c}}\right) \omega_{2} / k T\right] \exp \left[\xi\left(x_{1}^{\mathrm{s}}\right)^{2}\right]
\end{aligned}
$$

where $x_{1}^{\mathrm{s}}$ and $x_{2}^{\mathrm{s}}$ denote the respective molar fractions of components 1 and 2 in the two-component monolayers; $\pi_{1}^{\mathrm{c}}$ and $\pi_{2}^{\mathrm{c}}$ are the respective collapse pressures of components 1 and $2 ; \pi_{\mathrm{m}}^{\mathrm{c}}$ is the collapse pressure of the two-component monolayer at a given composition of $x_{1}^{\mathrm{s}}\left(\right.$ or $\left.x_{2}^{\mathrm{s}}\right) ; \omega_{1}$ and $\omega_{2}$ are the corresponding molecular areas at the collapse; and $\xi$ is the interaction parameter. The solid curve at higher surface pressures was drawn by adjusting $\xi$ in Eq. (1) to obtain the best fit for the experimental values of collapse pressures. At high surface pressures (Fig. 3), the DPPC/ $\mathrm{F} 8 \mathrm{H} 5 \mathrm{OH}$ system, which is characterized by the fluidizing effect, indicates a negative azeotropic type. In contrast, the $\mathrm{DPPC} / \mathrm{F} 8 \mathrm{Hm} \mathrm{OH}$ systems $(m \geq 7)$ are of the positive azeotropic type in the small $X_{F 8 \mathrm{Hm} \mathrm{OH}}$ region, indicating a stronger interaction between heterogeneous monolayers and reduced interactions with each other in the large $X_{F 8 \mathrm{HmOH}}$ region.

The interaction energy can be calculated as follows:

$$
\Delta \varepsilon=\xi R T / z
$$

where $z$ is the number of nearest neighbors, equal to 6 , in a close-packed monolayer, and the interaction energy is $\Delta \varepsilon$ $=\varepsilon_{12}-\left(\varepsilon_{11}+\varepsilon_{22}\right) / 2^{40)} ; \varepsilon_{i j}$ denotes the potential energy of the interaction between components $i$ and $j$. Thus, the interaction between heterogeneous molecules is stronger for $\Delta \varepsilon<$ 0 than that between homogeneous molecules and vice versa. In the present systems, DPPC preferentially interacts with $\mathrm{F} 8 \mathrm{HmOH}(m \geq 7)$ rather than with $\mathrm{F} 8 \mathrm{H} 5 \mathrm{OH}$ in the close-packed monolayer state.

\section{Additional effect of $\mathbf{F 8 H m O H}$ on DPPC monolayers}

The $\pi-A$ and $\Delta V-A$ isotherms of DPPC monolayers containing $0-20$ wt $\% F 8 H 5 \mathrm{OH}$ and $F 8 H 11 \mathrm{OH}$ are shown in Fig. $4(\mathrm{~A})$ and $4(\mathrm{~B})$, respectively. The $\pi-A$ isotherm of DPPC monolayers moves to larger molecular areas with increasing amounts of $F 8 H 50 H$ (Fig. $4(\mathrm{~A})$ ). The $\pi^{\text {eq }}$ value corresponding to the disordered/ordered phase transition increases from $\sim 11$ to $\sim 14 \mathrm{mN} \mathrm{m}^{-1}$. As described above, this increase demonstrates that fluidization of the DPPC monolayers was induced by $\mathrm{F} 8 \mathrm{H} 5 \mathrm{OH}$ incorporation. The maximum $\Delta V$ values in the close-packed state decreased from $\sim 550$ (curve 1 ) to $\sim 370 \mathrm{mV}$ (curve 5 ) as the percentage of $\mathrm{F} 8 \mathrm{H} 5 \mathrm{OH}$ was increased. The negative variation resulted from the contribution of the fluorocarbon moiety in $F 8 H 5 O H$ molecules. In contrast, the $\pi^{\text {eq }}$ value for the DPPC/F8H11OH system (Fig. 4(B)) decreased from $\sim 11$ to $\sim 5 \mathrm{mN} \mathrm{m}^{-1}$ with increasing $F 8 H 11 \mathrm{OH}$ concentration, 

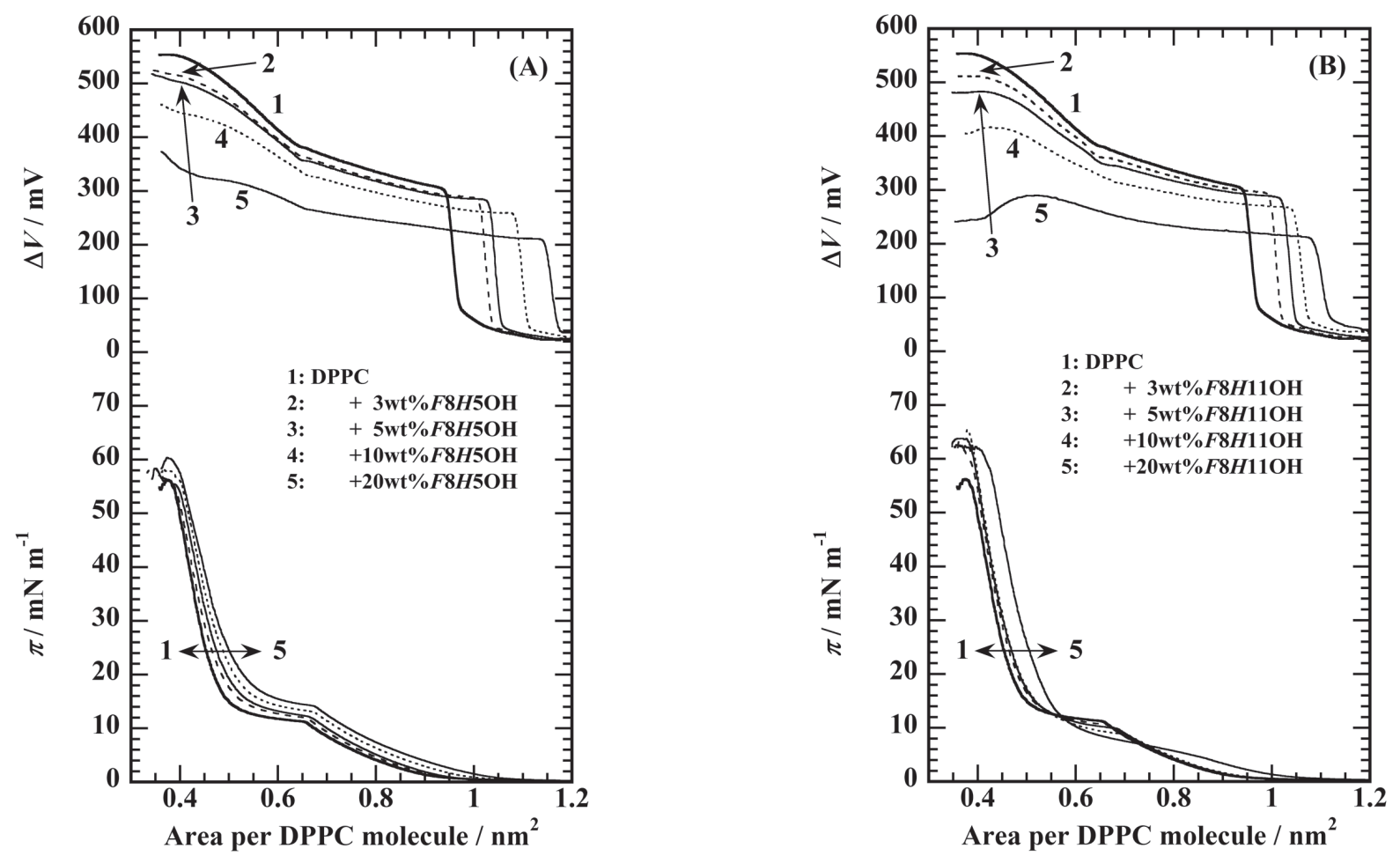

Fig. 4 The $\pi-A$ and $\Delta V-A$ isotherms of monolayers containing DPPC, plus 0 to $20 \mathrm{wt} \%$ of (A) $F 8 H 5 \mathrm{OH}$ and (B) $F 8 H 11 \mathrm{OH}$ on a $0.02 \mathrm{M}$ Tris buffer solution ( $\mathrm{pH} 7.4$ ) with $0.13 \mathrm{M} \mathrm{NaCl}$ at $298.2 \mathrm{~K}$. Reprinted with permission from ref. 27. Copyright (2015) American Chemical Society.

indicating the solidification of DPPC monolayers. Accordingly, the $\pi^{\mathrm{c}}$ value increased from 55 to $\sim 65 \mathrm{mN} \mathrm{m}^{-1}$. The magnitude of the decrease in the maximum $\Delta V$ value was larger compared to in the $F 8 \mathrm{H} 5 \mathrm{OH}$ system (Fig. 4(A)). This occurred because $F 8 H 110 H$ forms more ordered monolayer than $F 8 H 5 \mathrm{OH}$ because of its longer hydrophobic chain ${ }^{27)}$.

Figure 5 shows fluorescence microscopy (FM) images acquired in situ at the air-water interface for the DPPC monolayers with 0,5 , and 10 wt $\% F 8 H 5 \mathrm{OH}$ or $F 8 H 11 \mathrm{OH}$. Bright and dark regions in the FM images correspond respectively to the disordered and ordered phases of monolayers, as the fluorescent probes (here, NBD-PC) selectively dissolve into the disordered phases. The FM images of DPPC monolayers below the LE/LC transition are homogeneously bright. As shown in Fig. 5(a), nucleation of the LC domains occurs beyond the transition and the ordered domain increases in size upon compression. The ordered domain with counterclockwise arms is characteristic of $\operatorname{DPPC}(L$ type $)$ monolayers ${ }^{42,43}$. The addition of $5 \mathrm{wt} \%$ $F 8 H 5 \mathrm{OH}$ to DPPC causes the arms of the domains to become slim and branched (Fig. 5(b)). This morphological variation is reinforced by further addition of $\mathrm{F} 8 \mathrm{H} 5 \mathrm{OH}$ (Fig. 5 (c)). The ordered domain shape is commonly controlled by the line tension of the phase boundary and long-range dipole interactions ${ }^{44-50)}$. The FM phase behavior in the
$\mathrm{DPPC} / F 8 H 5 \mathrm{OH}$ systems is dominated by long-range dipoledipole interactions ${ }^{27)}$. That is, $F 8 H 5 \mathrm{OH}$ decreases the width and increases the length of the arms in the ordered domain by reducing line tensions between the ordered and disordered phases. Similar dramatic alternations in domain shapes were reported for DPPC/cholesterol systems ${ }^{51,52)}$. Therefore, $F 8 H 5 \mathrm{OH}$ may have similar properties to cholesterol, the role of which remains controversial in human pulmonary surfactants. Nevertheless, $F 8 H 5 \mathrm{OH}$ may decrease line tension and lower surface viscosity induced by the DPPC: $F 8 H 5 \mathrm{OH}$ complex ${ }^{52)}$. The incorporation of 5 wt $\% \mathrm{~F} 8 \mathrm{H} 11 \mathrm{OH}$ causes the arm of ordered domains to increase in width in contrast to the $\mathrm{F} 8 \mathrm{H} 5 \mathrm{OH}$ cases (Fig. 5 (d), (e)). Thus, $F 8 H 110 \mathrm{H}$ considerably changes the line tension of the phase boundary to have positive values. The larger size of the ordered domains strongly supports the solidifying effect of $F 8 H 11 \mathrm{OH}$ and the notion that a preferential interaction occurs between DPPC and $F 8 H 110 H$.

\section{Additional effect of $\mathrm{F} 8 \mathrm{HmOH}$ on model PS monolayers}

The binary DPPC/Hel 13-5 monolayer fixed at $X_{\text {Hel 13-5 }}=$ 0.1 was used as a simple model mixture of synthetic pulmonary surfactants ${ }^{28,30,31}$. This monolayer showed a disor- 
Fig. 5 FM images of monolayers of (a) DPPC alone, (b) DPPC plus $5 \mathrm{wt} \%$ of $F 8 H 5 \mathrm{OH}$, (c) $10 \mathrm{wt} \%$ of $F 8 H 5 \mathrm{OH}$, (d) $5 \mathrm{wt} \%$ of $F 8 H 11 \mathrm{OH}$, (e) $10 \mathrm{wt} \%$ of $F 8 H 11 \mathrm{OH}$ on a $0.02 \mathrm{M}$ Tris buffer solution (pH 7.4) with $0.13 \mathrm{M} \mathrm{NaCl}$ at $298.2 \mathrm{~K}$. Numbers within open circles indicate the surface pressure $\left(\mathrm{mN} \mathrm{m}^{-1}\right)$. The monolayers contain $1 \mathrm{~mol} \%$ of the fluorescent probe (NBD-PC). The scale bar in the lower right represents $100 \mu \mathrm{m}$. Reprinted with permission from ref. 27. Copyright (2015) American Chemical Society.

(a)
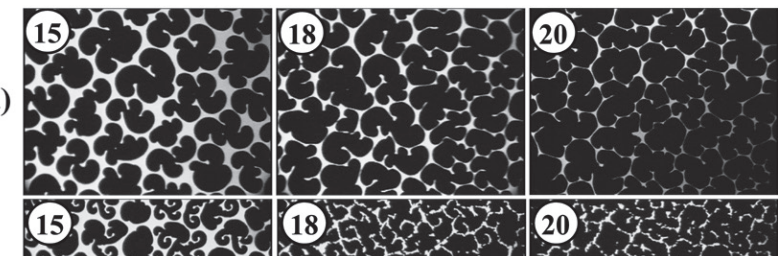

(b)

(c)
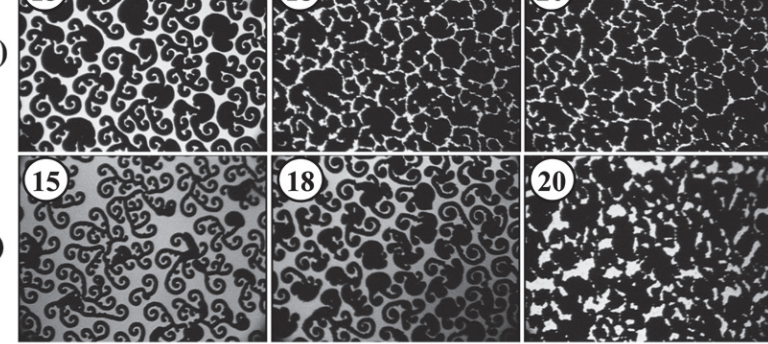

(d)
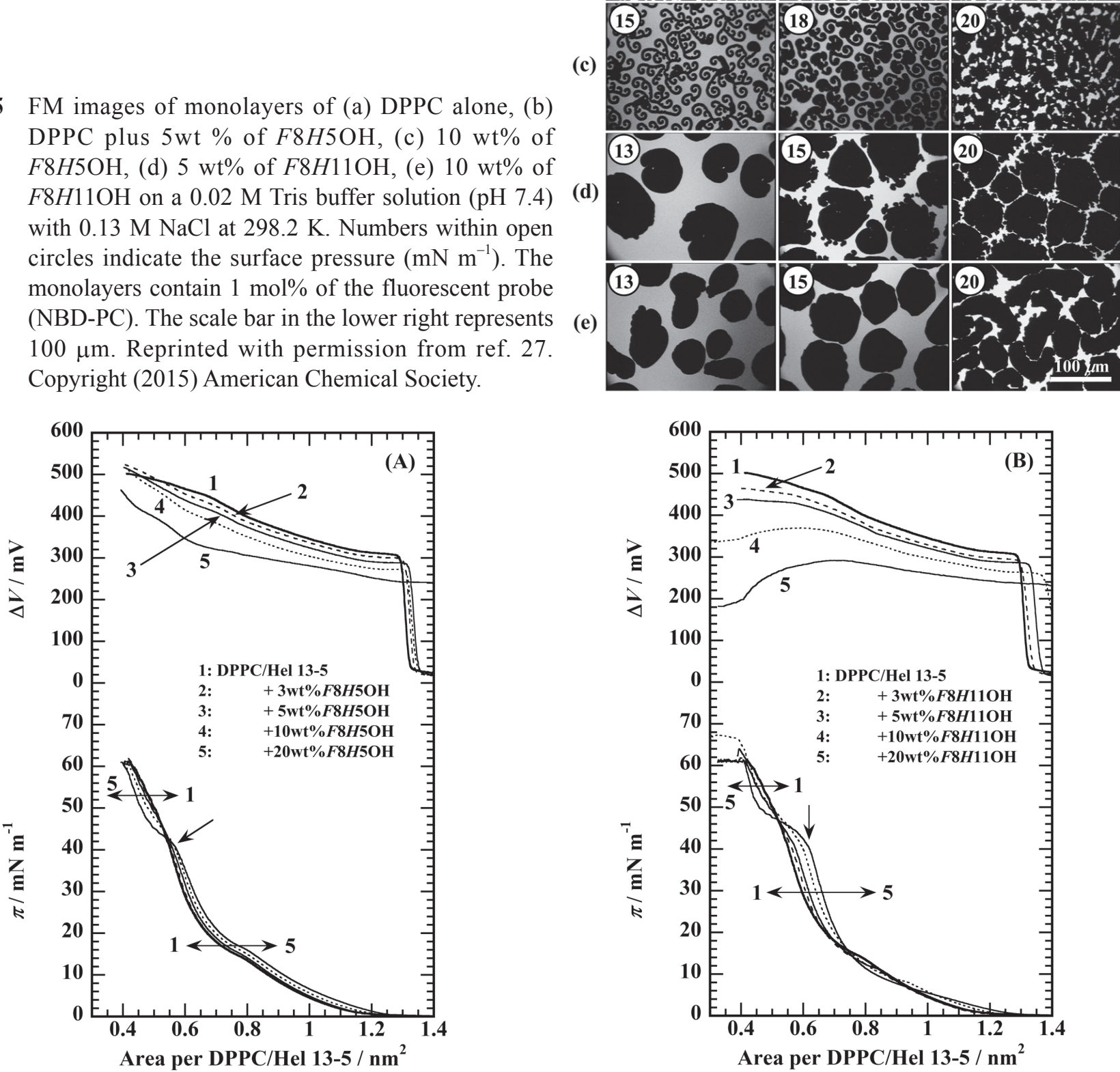

Fig. 6 The $\pi-A$ and $\Delta V-A$ isotherms of monolayers containing the model PS preparation (or the binary DPPC/Hel 13-5 mixture with the fixed ratio of $X_{\text {Hel 13-5 }}=0.1$ ) plus 0 to $20 \mathrm{wt} \%$ of (A) $F 8 H 5 \mathrm{OH}$ and (B) $F 8 H 11 \mathrm{OH}$ on a 0.02 M Tris buffer solution ( $\mathrm{pH} 7.4$ ) with $0.13 \mathrm{M} \mathrm{NaCl}$ at $298.2 \mathrm{~K}$. Reprinted with permission from ref. 27. Copyright (2015) American Chemical Society.

dered/ordered phase transition at $\sim 14 \mathrm{mN} \mathrm{m}^{-1}$ and plateau at $\sim 42 \mathrm{mN} \mathrm{m}^{-1}$, where Hel 13-5 begins to be squeezed out of DPPC monolayers. The squeezing-out behavior is very important for pulmonary functions during 
respiration $^{28,53-55)}$. Indeed, the squeezed-out phenomenon observed for $\pi-A$ isotherms at $\sim 42 \mathrm{mN} \mathrm{m}^{-1}$ is closely related to reducing the work of breathing at the alveolar surface. Below the plateau pressure, the $\pi-A$ isotherm shifts slightly to larger molecular areas with increasing amounts of $\mathrm{F} 8 \mathrm{H} 5 \mathrm{OH}$ (Fig. $6(\mathrm{~A})$ ). Accordingly, the plateau region (or plateau length) becomes wider, suggesting the improvement of the squeezed-out action of Hel 13-5 upon compression. This is also supported by the shift of the $\pi-A$ isotherm toward smaller molecular areas above the plateau pressure. The maximum $\Delta V$ values of $\sim 500 \mathrm{mV}$ for the preparations with $3-10 \mathrm{wt} \% \mathrm{~F} 8 \mathrm{H} 5 \mathrm{OH}$ are similar to that of the DPPC/Hel 13-5 mixture. This indicates that $\mathrm{F} 8 \mathrm{H} 5 \mathrm{OH}$ is also squeezed out of the surface together with Hel 13-5 upon compression. For the mixture containing $20 \mathrm{wt} \%$ $F 8 H 5 \mathrm{OH}$, excess $\mathrm{F} 8 \mathrm{H} 5 \mathrm{OH}$ remaining at the surface contributes negatively to the $\Delta V$ value $^{27)}$. In contrast, at intermediate surface pressures, $\pi-A$ isotherms for the $F 8 H 110 H$ system clearly move to larger molecular areas with increasing $\mathrm{F} 8 \mathrm{H} 11 \mathrm{OH}$ concentration (Fig. 6(B)). Moreover, the plateau region is longer than that of the $\mathrm{F} 8 \mathrm{H} 5 \mathrm{OH}$ systems. The $\Delta V$ value decreases in the close-packed state depending on the $F 8 H 11 \mathrm{OH}$ levels, suggesting that $\mathrm{F} 8 \mathrm{H} 11 \mathrm{OH}$ molecules remain just at the surface above the plateau regions in contrast to $\mathrm{F} 8 \mathrm{H} 5 \mathrm{OH}$. Considering the monolayer miscibility between DPPC and $F 8 H 11 \mathrm{OH}$, the packing or orientation of DPPC monolayers is improved by $F 8 H 11 \mathrm{OH}$ such that the resultant rigid monolayer promotes the squeezingout of Hel 13-5 from the interface at high surface pressures.

Figure 7 shows the influence of $F 8 H m \mathrm{OH}$ addition on the FM morphology of the DPPC/Hel 13-5 monolayers. In Fig. $7(a)$, the ordered domain of DPPC monolayers is exhibited by a dark contrast, as Hel 13-5 itself forms a homogeneous disordered (bright) phase ${ }^{30}$. The ordered domains change in appearance compared to monolayers of DPPC alone. This is induced by lateral interactions between DPPC and Hel 13-5. Incorporation of $5 \mathrm{wt} \% \mathrm{~F} 8 \mathrm{H} 5 \mathrm{OH}$ fluidizes the ordered domain (Fig. $7($ b)). However, the size of the ordered domains increases with further addition of F8H5OH (Fig. 7 (c)). Interestingly, the appearance of the ordered domain is quite similar to that of pure DPPC monolayers (Fig. 5(a)). F8H5OH interacts more preferably with Hel 13-5 than with DPPC. The $F 8 H 110 H$ systems (Fig. $7(\mathrm{~d}),(\mathrm{e})$ ) indicate that the phase behavior and variation are similar to those in DPPC/F8H11OH systems (Fig. 5(d), (e)). That is, $F 8 H 110 H$ interacts favorably with the ordered domain of DPPC monolayers rather than with Hel $13-5$.

\section{Hysteresis behavior of the model PS monolayers}

Cycling $\pi-A$ and $\Delta V-A$ isotherms, which simulate respi- (a)
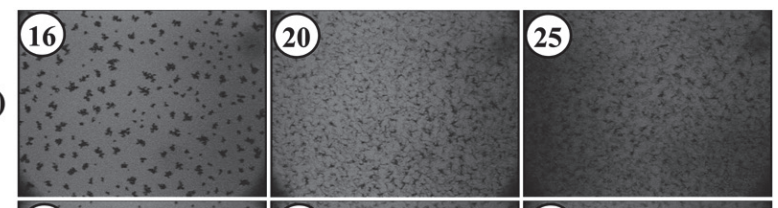

(b)
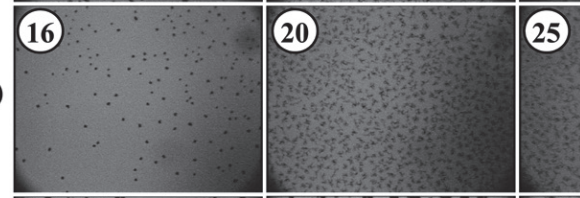

(c)
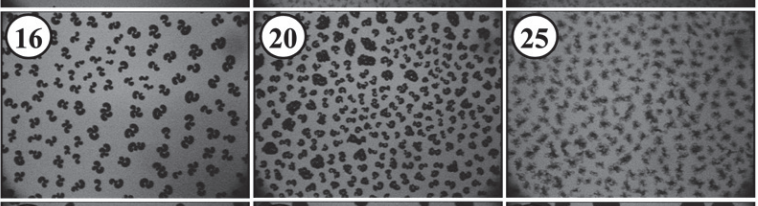

(d)
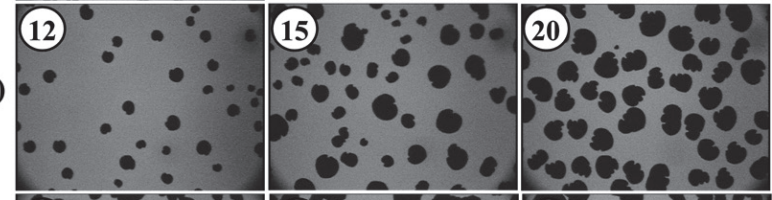

(e)
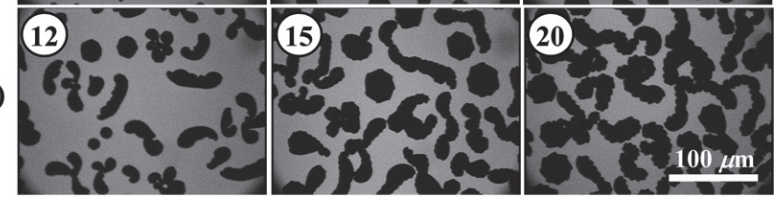

Fig. 7 FM images of monolayers of (a) the binary DPPC/Hel 13-5 mixture $\left(X_{\mathrm{Hel} \mathrm{13-5}}=0.1\right)$, (b) plus $5 \mathrm{wt} \%$ of $\mathrm{F} 8 \mathrm{H} 5 \mathrm{OH}$, (c) $10 \mathrm{wt} \%$ of $\mathrm{F} 8 \mathrm{H} 5 \mathrm{OH}$, (d) $5 \mathrm{wt} \%$ of $F 8 H 11 \mathrm{OH}$, (e) $10 \mathrm{wt} \%$ of $F 8 H 11 \mathrm{OH}$ on a $0.02 \mathrm{M}$ Tris buffer solution $(\mathrm{pH} 7.4)$ with $0.13 \mathrm{M} \mathrm{NaCl}$ at 298.2 K. Numbers within open circles indicate the surface pressure $\left(\mathrm{mN} \mathrm{m}^{-1}\right)$. The monolayers contain 1 mol\% of the fluorescent probe (NBD-PC). The scale bar in the lower right represents $100 \mu \mathrm{m}$. Reprinted with permission from ref. 27. Copyright (2015) American Chemical Society.

ratory movement in mammalian alveoli, were measured for the present systems. Indeed, the monolayer was compressed up to the respective collapse pressure and then expanded to the starting molecular areas. This process was successively repeated to determine the reproducibility of the respreading process within a monolayer. The $\pi-A$ and $\Delta V-A$ isotherms for the DPPC/Hel 13-5 system without $\mathrm{F} 8 \mathrm{Hm} \mathrm{OH}$ indicate good reproducibility among all rounds and showed a hysteresis loop, which is enclosed between isotherms upon compression and successive expansion stages $^{27)}$. The hysteresis loops for the $20 \mathrm{wt} \% \mathrm{~F} 8 \mathrm{H} 5 \mathrm{OH}$ (Fig. 8(A)) and F8H11OH (Fig. 8(B)) additional system are debilitated with increasing cycling number. Interestingly, there is a considerable difference in $\Delta V$ behavior for both systems. For the $F 8 H 5 \mathrm{OH}$ system, $\Delta V$ values in the closepacked state of $A=\sim 0.4 \mathrm{~nm}^{2}$ increase as cycling number increases. This increase in $\Delta V$ suggests that $F 8 H 5 O H$ is 


\section{H. Nakahara}
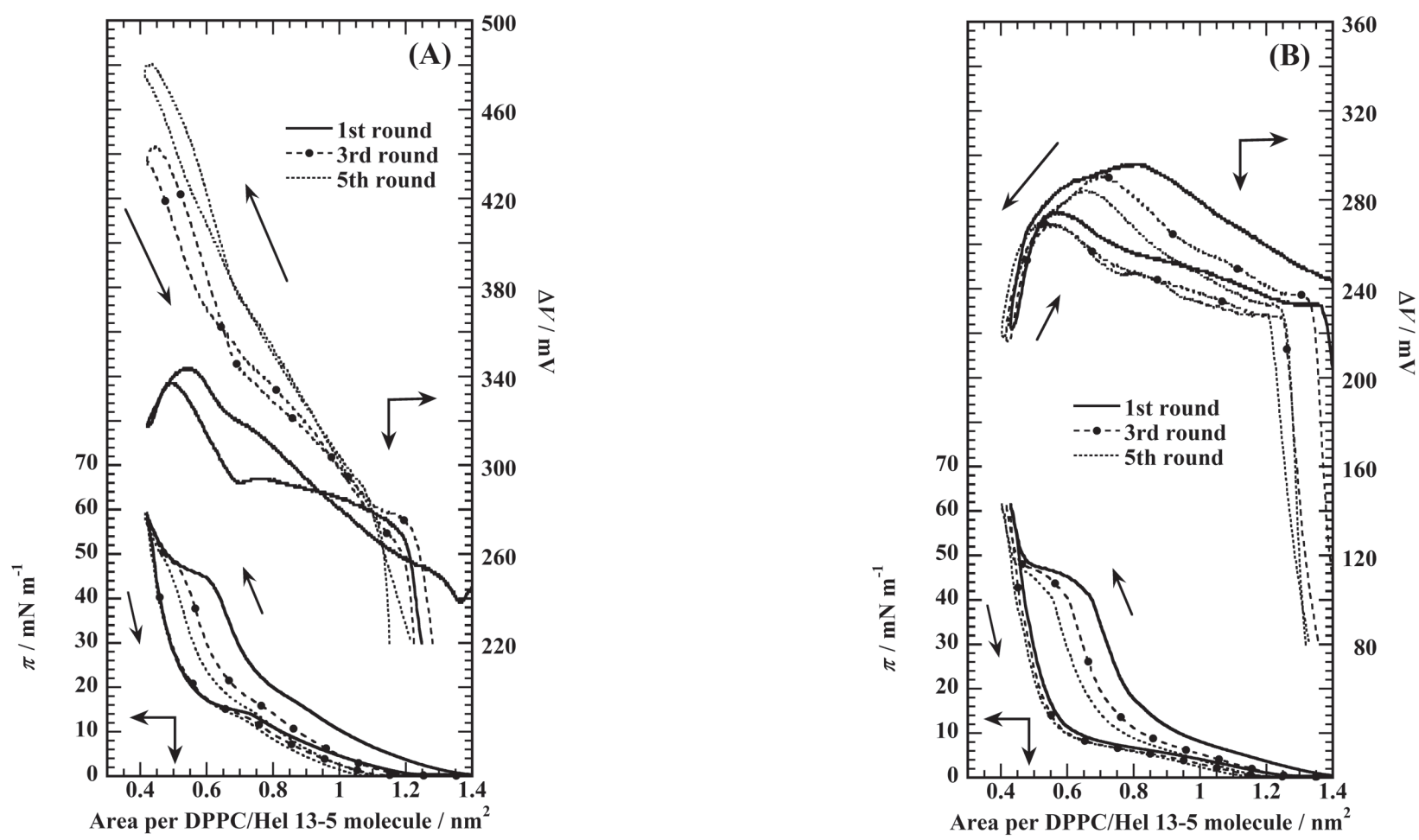

Fig. 8 Cyclic compression and expansion (or hysteresis curves) for surface pressures and surface potentials of monolayers containing the DPPC/Hel $13-5$ preparation with (A) $20 \mathrm{wt} \%$ of $F 8 H 5 \mathrm{OH}$ and (B) of $F 8 H 11 \mathrm{OH}$. The subphase was a $0.02 \mathrm{M}$ Tris buffer solution $(\mathrm{pH} 7.4)$ with $0.13 \mathrm{M} \mathrm{NaCl}$ at $298.2 \mathrm{~K}$. The compression-expansion cycle was performed five times at the compression rate of $\sim 0.25 \mathrm{~nm}^{2}$ molecule ${ }^{-1} \mathrm{~min}^{-1}$.
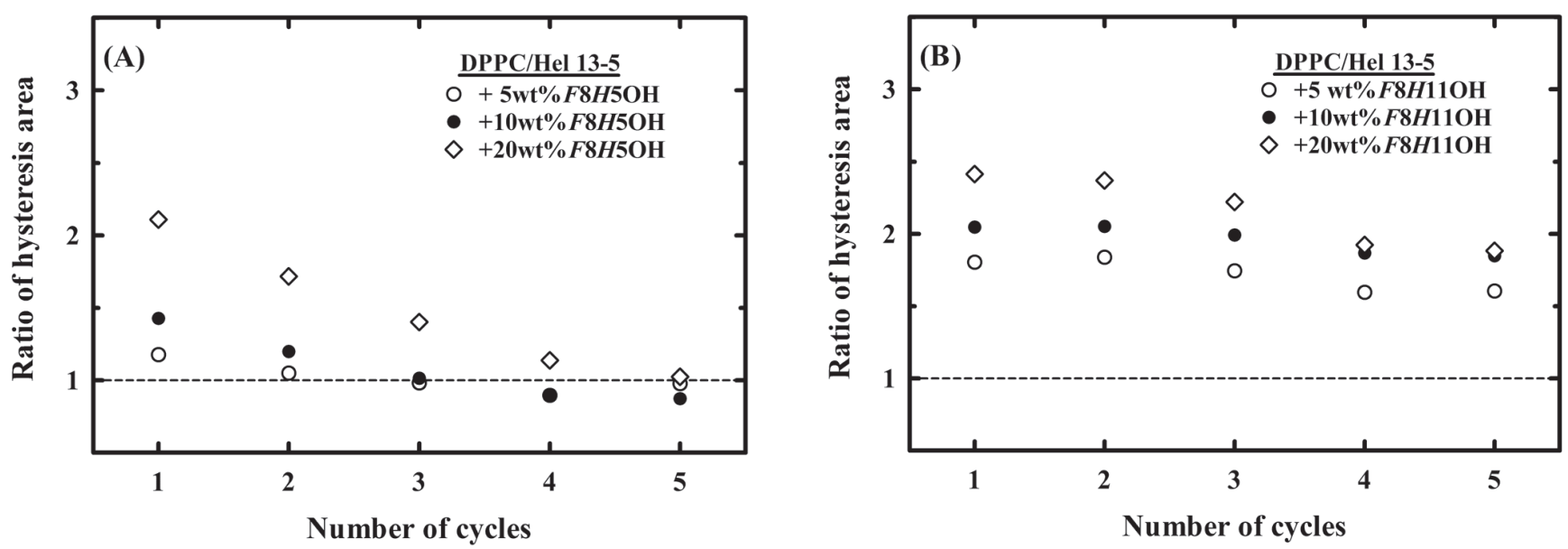

Fig. 9 Ratio of the integrated hysteresis area between compression and expansion isotherms for the DPPC/Hel 13-5 preparation $\left(X_{\mathrm{Hel} \mathrm{13-5}}=0.1\right.$ ) containing (A) $\mathrm{F} 8 \mathrm{H5OH}$ and (B) $\mathrm{F} 8 \mathrm{H} 11 \mathrm{OH}$ relative to that for the preparation without the alcohols as a function of the number of compression/expansion cycles. Adapted with permission from ref. 27. Copyright (2015) American Chemical Society.

gradually excluded through an irreversible process from the interface by the cycling ${ }^{27)}$. In contrast, in the $F 8 H 11 \mathrm{OH}$ system (Fig. $8(\mathrm{~B}))$, the $\Delta V$ value in each round in the close-packed state converges at $\sim 220 \mathrm{mV}$, indicating that $F 8 H 110 H$ molecules remain at the interface together with DPPC during the cycling.
The integrated hysteresis area based on successive compression-expansion $\pi-A$ isotherms has been quantitatively evaluated. This analysis can assess the reduction in work of breathing in the lung ${ }^{56-58)}$. Shown in Fig. 9 are plots of the ratio of integrated hysteresis area of the cyclic $\pi-A$ isotherm for the DPPC/Hel 13-5 mixture containing 5-10 
wt $\% F 8 H m \mathrm{OH}$ to that for the mixture without $\mathrm{F} 8 \mathrm{HmOH}$ as a function of cycling numbers. The ratio of hysteresis area for the $\mathrm{F} 8 \mathrm{H} 5 \mathrm{OH}$ systems (Fig. 9(A)) decreases with increasing cycling number and finally converges on unity. This result also supports the irreversible elimination of F8H5OH together with Hel 13-5 from the surface monolayer. In contrast, the $F 8 H 110 H$ systems show a slight decrease in the ratio with respect to the number of cycles (Fig. 9(B)). However, the ratio was maintained at more than 1.5 even in the fifth round as opposed to that observed in the $F 8 H 5 \mathrm{OH}$ systems, supporting the solidifying effect of $F 8 H 11 \mathrm{OH}$ at the surface. Additionally, the effectiveness of the PS model preparation is improved by incorporation of 5-10 wt\% F8H11OH.

\section{Conclusions and perspectives}

Perfluorooctylated long-chain fatty alcohols $(F 8 \mathrm{HmOH}$, $m=5,7,9$, and 11) have been characterized employing the monolayer technique at the air-water interface as an additive for PS preparations. All $F 8 \mathrm{HmOH}$ molecules described here are miscible with DPPC as the major component in native PS within a monolayer. However, completely different interaction modes are observed among binary DPPC/ $\mathrm{F} 8 \mathrm{Hm} \mathrm{OH}$ systems; a negative azeotrope for DPPC/F8H5OH and positive azeotrope for the others. FM observations showed that $F 8 \mathrm{HmOH}$ exerts fluidizing (for $m=5$ ) or solidifying (for $m=7-11$ ) effects on DPPC monolayers depending on the hydrophobic chain length of $F 8 \mathrm{HmOH}$. These effects are also observed in the model PS preparation (DPPC/Hel 13-5) incorporated with a small amount of $F 8 H 5 \mathrm{OH}$ or $F 8 H 11 \mathrm{OH}$. The evaluation of $\pi$ - $A$ isotherms measured under the successive compression-expansion reveals the molecular action of $F 8 H 5 \mathrm{OH}$ and $F 8 \mathrm{H} 11 \mathrm{OH}$ along the squeeze-out phenomenon of Hel 13-5; $F 8 H 5 \mathrm{OH}$ undergoes irreversible exclusion from the surface upon compression, whereas $F 8 H 11 \mathrm{OH}$ enhances the squeezingout behavior of Hel 13-5 by forming a solid-like monolayer with DPPC at high surface pressures. For NRDS therapy, partially fluorinated compounds may be useful as additives to PS formulations, which require further fundamental and clinical investigations.

\section{Acknowledgements}

I am deeply grateful to Dr. Osamu Shibata and Dr. Sannamu Lee, Nagasaki International University for helpful discussions.

\section{References}

1) Krafft, M. P.; Riess, J. G. Chemistry, physical chemistry, and uses of molecular fluorocarbon-hydrocarbon diblocks, triblocks, and related compounds--unique "apolar" components for self-assembled colloid and interface engineering. Chem. Rev. 109, 1714-1792 (2009).

2) Riess, J. G. Fluorous micro- and nanophases with a biomedical perspective. Tetrahedron 58, 4113-4131 (2002).

3) Miller, T. M.; Bederson, B. Atomic and molecular polarizabilities-a review of recent advances. Adv. Atom. Mol. Phys. 13, 1-55 (1978).

4) Dunitz, J. D. Organic fluorine: odd man out. ChemBioChem 5, 614-621(2004).

5) Krafft, M. P.; Riess, J. G. Highly fluorinated amphiphiles and colloidal systems, and their applications in the biomedical field. A contribution. Biochimie 80, 489514 (1998)

6) Krafft, M. P.; Rolland, J. P.; Vierling, P.; Riess, J. G. New perfluoroalkylated phosphocholines. Effect on particle size and stability of fluorocarbon emulsions. New J. Chem. 14, 869-875 (1990).

7) Sadtler, V. M.; Jeanneaux, F.; Pierre Krafft, M.; Rabai, J.; Riess, J. G. Perfluoroalkylated amphiphiles with a morpholinophosphate or a dimorpholinophosphate polar head group. New J. Chem. 22, 609-613(1998).

8) Broniatowski, M.; Nieto-Suarez, M.; Vila-Romeu, N.; Hac-Wydro, K.; Dynarowicz-Łatka, P. Two-dimensional miscibility between a semifluorinated hydrocarbon and hydrogenated alcohols. Colloids Surf. A 249, 3-9 (2004).

9) Broniatowski, M.; Sandez Macho, I.; Miñnes, J.,Jr.; Dynarowicz-Łatka, P. Langmuir Monolayers Characteristic of(Perfluorodecyl)-Alkanes. J. Phys. Chem. B 108, 13403-13411 (2004).

10) Ren, Y.; Shoichet, M. S.; McCarthy, T. J.; Hsu, S. L. Spectroscopic characterization of polymer adsorption at the air-solution interface. Macromolecules 28, 358364 (1995).

11) Nakahara, H.; Tsuji, M.; Sato, Y.; Krafft, M. P.; Shibata, O. Langmuir monolayer miscibility of single-chain partially fluorinated amphiphiles with tetradecanoic acid. J. Colloid Interface Sci. 337, 201-210 (2009).

12) Nakamura, S.; Nakahara, H.; Krafft, M. P.; Shibata, O. Two-component Langmuir monolayers of single-chain partially fluorinated amphiphiles with dipalmitoylphosphatidylcholine (DPPC) . Langmuir 23, 12634-12644 (2007).

13) Lehmler, H.-J.; Bummer, P. M. Mixing behavior of 10 (perfluorohexyl)-decanol and DPPC. Colloids Surf. B 44, 74-81 (2005).

14) Shibata, O.; Krafft, M. P. Mixed Langmuir monolayers made from single-chain perfluoroalkylated amphiphi- 


\section{H. Nakahara}

les. Langmuir 16, 10281-10286 (2000).

15) Krüger, P.; Baatz, J. E.; Dluhy, R. A.; Lösche, M. Effect of hydrophobic surfactant protein SP-C on binary phospholipid monolayers. Molecular machinery at the air/water interface. Biophys. Chem. 99, 209-228 (2002).

16) Veldhuizen, R.; Nag, K.; Orgeig, S.; Possmayer, F. The role of lipids in pulmonary surfactant. Biochim. Biophys. Acta 1408, 90-108(1998).

17) Pérez-Gil, J.; Keough, K. M. W. Interfacial properties of surfactant proteins. Biochim. Biophys. Acta 1408, 203-217 (1998).

18) Yu, S.-H.; Possmayer, F. Lipid compositional analysis of pulmonary surfactant monolayers and monolayer-associated reservoirs. J. Lipid Res. 44, 621-629(2003).

19) Pérez-Gil, J. Structure of pulmonary surfactant membranes and films: the role of proteins and lipid-protein interactions. Biochim. Biophys. Acta 1778, 16761695 (2008).

20) Cochrane, C. G. Pulmonary surfactant in allergic inflammation: New insights into the molecular mechanisms of surfactant function. Am. J. Physiol. 288, L608-L609 (2005).

21) Batenburg, J. J.; Haagsman, H. P. The lipids of pulmonary surfactant: dynamics and interactions with proteins. Prog. Lipid Res. 37, 235-276(1998).

22) Notter, R. H., Lung surfactants: Basic science and clinical applications. 149; Ed.; Marcel Dekker, Inc.: New York, Basel, pp.1-444(2000).

23) Nakahara, H.; Lee, S.; Sugihara, G.; Chang, C.-H.; Shibata, O. Langmuir monolayer of artificial pulmonary surfactant mixtures with an amphiphilic peptide at the air/water interface: Comparison of new preparations with Surfacten (Surfactant TA). Langmuir 24, 33703379 (2008).

24) Wiswell, T. E.; Smith, R. M.; Katz, L. B.; Mastroianni, L.; Wong, D. Y.; Willms, D.; Heard, S.; Wilson, M.; Hite, R. D.; Anzueto, A.; Revak, S. D.; Cochrane, C. G. Bronchopulmonary segmental lavage with Surfaxin (KL(4) -surfactant) for acute respiratory distress syndrome. Am. J. Respir. Crit. Care Med. 160, 1188-1195 (1999).

25) Ma, J.; Koppenol, S.; Yu, H.; Zografi, G. Effects of a cationic and hydrophobic peptide, KL4, on model lung surfactant lipid monolayers. Biophys. J. 74, 18991907 (1998).

26) Revak, S. D.; Merritt, T. A.; Cochrane, C. G.; Heldt, G. P.; Alberts, M. S.; Anderson, D. W.; Kheiter, A. Efficacy of synthetic peptide-containing surfactant in the treatment of respiratory distress syndrome in preterm infant rhesus monkeys. Pediatr. Res. 39, 715-724 (1996).

27) Nakahara, H.; Lee, S.; Krafft, M. P.; Shibata, O. Fluorocarbon-hybrid pulmonary surfactants for replacement therapy - a Langmuir monolayer study. Langmuir 26, 18256-18265(2010).

28) Nakahara, H.; Lee, S.; Shibata, O. Pulmonary surfactant model systems catch the specific interaction of an amphiphilic peptide with anionic phospholipid. Biophys. J. 96, 1415-1429(2009).

29) Nakahara, H.; Lee, S.; Sugihara, G.; Shibata, O. Mode of interaction of hydrophobic amphiphilic a-helical peptide/dipalmitoylphosphatidylcholine with phosphatidylglycerol or palmitic acid at the air-water interface. Langmuir 22, 5792-5803 (2006).

30) Nakahara, H.; Nakamura, S.; Hiranita, T.; Kawasaki, H.; Lee, S.; Sugihara, G.; Shibata, O. Mode of interaction of amphiphilic $\alpha$-helical peptide with phosphatidylcholines at the air-water interface. Langmuir 22, 1182 $1192(2006)$.

31) Nakahara, H.; Lee, S.; Shibata, O. Specific interaction restrains structural transitions of an amphiphilic peptide in pulmonary surfactant model systems: An in situ PM-IRRAS investigation. Biochim. Biophys. Acta 1798, 1263-1271 (2010).

32) Nakamura, Y.; Yukitake, K.; Nakahara, H.; Lee, S.; Shibata, O.; Lee, S. Improvement of pulmonary surfactant activity by introducing D-amino acids into highly hydrophobic amphiphilic a-peptide Hel 13-5. Biochim. Biophys. Acta 1838, 2046-2052(2014).

33) Nakahara, H.; Lee, S.; Shibata, O. Surface pressure induced structural transitions of an amphiphilic peptide in pulmonary surfactant systems by an in situ PM-IRRAS study. Biochim. Biophys. Acta 1828, 1205-1213 (2013).

34) Lee, S.; Furuya, T.; Kiyota, T.; Takami, N.; Murata, K.; Niidome, Y.; Bredesen, D. E.; Ellerby, H. M.; Sugihara, G. De novo-designed peptide transforms Golgi-specific lipids into Golgi-like nanotubules. J. Biol. Chem. 276, 41224-41228(2001).

35) Furuya, T.; Kiyota, T.; Lee, S.; Inoue, T.; Sugihara, G.; Logvinova, A.; Goldsmith, P.; Ellerby, H.M. Nanotubules formed by highly hydrophobic amphiphilic a-helical peptides and natural phospholipids. Biophys. J. 84, 1950-1959 (2003).

36) Kiyota, T.; Lee, S.; Sugihara, G. Design and synthesis of amphiphilic a-helical model peptides with systematically varied hydrophobic-hydrophilic balance and their interaction with lipid- and bio-membranes. Biochemistry 35, 13196-13204(1996).

37) Gerber, F.; Krafft Marie, P.; Vandamme Thierry, F.; Goldmann, M.; Fontaine, P. Fluidization of a dipalmitoyl phosphatidylcholine monolayer by fluorocarbon gases: potential use in lung surfactant therapy. Biophys. J. 90, 3184-3192(2006).

38) Gerber, F.; Krafft, M. P.; Vandamme, T. F. The detrimental effect of serum albumin on the re-spreading of a dipalmitoyl phosphatidylcholine Langmuir monolay- 
er is counteracted by a fluorocarbon gas. Biochim. Biophys. Acta 1768, 490-494(2007).

39) Nakahara, H.; Nakamura, S.; Okahashi, Y.; Kitaguchi, D.; Kawabata, N.; Sakamoto, S.; Shibata, O. Examination of fluorination effect on physical properties of saturated long-chain alcohols by DSC and Langmuir monolayer. Colloids Surf. B 102, 472-478(2013).

40) Joos, P.; Demel, R. A. The interaction energies of cholesterol and lecithin in spread mixed monolayers at the air-water interface. Biochim. Biophys. Acta 183, 447-457 (1969).

41) Savva, M.; Acheampong, S. The interaction energies of cholesterol and 1,2-dioleoyl-sn-glycero-3-phosphoethanolamine in spread mixed monolayers at the air-water interface. J. Phys. Chem. B 113, 9811-9820 (2009).

42) Weis, R. M.; McConnell, H. M. Two-dimensional chiral crystals of phospholipid. Nature 310, 47-49 (1984).

43) Cruz, A.; Vázquez, L.; Vélez, M.; Pérez-Gil, J. Influence of a fluorescent probe on the nanostructure of phospholipid membranes: dipalmitoylphosphatidylcholine interfacial monolayers. Langmuir 21, 5349-5355 (2005).

44) Benvegnu, D. J.; McConnell, H. M. Surface dipole densities in lipid monolayers. J. Phys. Chem. 97, 66866691 (1993).

45) Benvegnu, D. J.; McConnell, H. M. Line tension between liquid domains in lipid monolayers. J. Phys. Chem. 96, 6820-6824(1992).

46) Moy, V. T.; Keller, D. J.; McConnell, H. M. Molecular order in finite two-dimensional crystals of lipid at the air-water interface. J. Phys. Chem. 92, 5233-5238 (1988).

47) Keller, D. J.; Korb, J. P.; McConnell, H. M. Theory of shape transitions in two-dimensional phospholipid domains. J. Phys. Chem. 91, 6417-6422 (1987).

48) Keller, D. J.; McConnell, H. M.; Moy, V. T. Theory of superstructures in lipid monolayer phase transitions. $J$. Phys. Chem. 90, 2311-2315(1986).

49) McConnell, H. M. Harmonic shape transitions in lipid monolayer domains. J. Phys. Chem. 94, 4728-4731 (1990).

50) McConnell, H. M. Structures and transitions in lipid monolayers at the air-water interface. Annu. Rev. Phys. Chem. 42, 171-195(1991).

51) Weis, R. M.; McConnell, H. M. Cholesterol stabilizes the crystal-liquid interface in phospholipid monolayers. J. Phys. Chem. 89, 4453-4459 (1985).

52) Kim, K.; Choi, S. Q.; Zell, Z. A.; Squires, T. M.; Zasadzinski, J. A. Effect of cholesterol nanodomains on monolayer morphology and dynamics. Proc. Natl. Acad. Sci. USA 110, E3054-E3060 (2013).

53) Alonso, C.; Alig, T.; Yoon, J.; Bringezu, F.; Warriner, H.; Zasadzinski, J. A. More than a monolayer: Relating lung surfactant structure and mechanics to composition. Biophys. J. 87, 4188-4202 (2004).

54） Diemel, R. V.; Snel, M. M. E.; Waring, A. J.; Walther, F. J.; van Golde, L. M. G.; Putz, G.; Haagsman, H. P.; Batenburg, J. J. Multilayer formation upon compression of surfactant monolayers depends on protein concentration as well as lipid composition. An atomic force microscopy study. J. Biol. Chem. 277, 21179-21188 (2002).

55) Takamoto, D. Y.; Lipp, M. M.; Von Nahmen, A.; Lee, K. Y. C.; Waring, A. J.; Zasadzinski, J. A. Interaction of lung surfactant proteins with anionic phospholipids. Biophys. J. 81, 153-169 (2001).

56) Davis, A. J.; Jobe, A. H.; Hafner, D.; Ikegami, M. Lung function in premature lambs and rabbits treated with a recombinant SP-C surfactant. Am. J. Respir. Crit. Care Med. 157, 553-559 (1998).

57) Ikegami, M.; Weaver, T. E.; Conkright, J. J.; Sly, P. D.; Ross, G. F.; Whitsett, J. A.; Glasser, S. W. Deficiency of SP-B reveals protective role of SP-C during oxygen lung injury. J. Appl. Physiol. 92, 519-526 (2002).

58) Xu, Y.; Kobayashi, T.; Cui, X.; Ohta, K.; Kabata, C.; Tashiro, K. Lung volumes and alveolar expansion pattern in immature rabbits treated with serum-diluted surfactant. J. Appl. Physiol. 97, 1408-1413(2004). 